PROCEEDINGS OF THE

AMERICAN MATHEMATICAL SOCIETY

Volume 136, Number 11, November 2008, Pages 4027-4032

S 0002-9939(08)09375-1

Article electronically published on June 9, 2008

\title{
AN OPEN MAPPING THEOREM FOR YOUNG MEASURES
}

\author{
HIROSHI TATEISHI
}

(Communicated by Richard C. Bradley)

\begin{abstract}
Ditor and Eifler consider the open mapping theorem for the probability spaces. Here we attempt to generalize the theorem to the spaces of Young measures.
\end{abstract}

\section{INTRODUCTION}

The classical open mapping theorem states that a bounded linear operator defined on some Banach space onto another Banach space is an open mapping. It is well recognized that this property does not necessarily hold if we restrict the domain and the range of the operator as the following example illuminates:

Example 1 (Ditor and Eifler [4]). Let $X=[0,1] \times\{0,1\}, Y=[0,1]$ and $\varphi: X \longrightarrow$ $Y$ be defined by $\varphi(t, i)=t$. Then $\varphi$ is a continuous, onto and open mapping. We consider a map $\pi: M^{b}(X) \longrightarrow M^{b}(Y)$ defined by

$$
\pi(\mu)(g)=\mu(g \circ \varphi): g \in C([0,1])
$$

where $M^{b}(X)$ and $M^{b}(Y)$ denote the spaces of bounded measures on $X$ and $Y$, respectively, and $C([0,1])$ denotes the space of real-valued continuous functions defined on $[0,1]$. Let $U(X)$ and $U(Y)$ be the unit balls of $M^{b}(X)$ and $M^{b}(Y)$, respectively. Then, the map $\pi$ restricted on $U(X)$ is not an open mapping with respect to the weak* topologies for both $M^{b}(X)$ and $M^{b}(Y)$. Indeed, let $\mu_{0}=$ $\frac{1}{2}\left(\delta_{(1,1)}-\delta_{(1,0)}\right), \delta_{a}$ denote a Dirac measure, the probability of which is concentrated at $a$. Then $\pi\left(\mu_{0}\right)=0$. Also, the following convergence holds true:

$$
\nu_{n}=\frac{1}{2}\left(\delta_{0}-\delta_{1 / n}\right) \longrightarrow 0 \text { weakly }^{*} \text { in } U(Y) .
$$

Since the support of the measure $\mu_{n} \in U(X)$ with $\pi\left(\mu_{n}\right)=\nu_{n}$ must be included in $\varphi^{-1}(\{0,1 / n\})$, such a sequence $\mu_{n}$ never converges weakly* to $\mu_{0}$.

Recall that the proof of the open mapping theorem rests on the Baire category theorem and the completeness of the range of $\pi$ is crucial to establish the openness of $\pi$. In the above example, the set of bounded measures with total variation 1 is not complete with respect to the weak* topology, and this fact induces the failure of the theorem. Also, it is conjectured that if the domain and the range of $\pi$ are restricted to the set of probability measures or the set of sub-probability measures,

Received by the editors December 29, 2006, and, in revised form, September 7, 2007, and October 2, 2007.

2000 Mathematics Subject Classification. Primary 60B05, 54C60.

Key words and phrases. Young measure, open mapping, narrow topology.

(C)2008 American Mathematical Society 
$\pi$ would be an open map. Ditor and Eifler 4 consider these cases. Let $\mathbb{S}, \mathbb{T}$ be compact metric spaces and denote by $P(\mathbb{S}), P(\mathbb{T})$ the spaces of Borel probability measures on $\mathbb{S}$ and $\mathbb{T}$ respectively. A continuous mapping $\varphi$ defined on $\mathbb{S}$ onto $\mathbb{T}$ is assumed to be given. For each probability measure $\mu \in P(\mathbb{S})$, we can define a probability measure $\pi(\mu)$ on $\mathbb{T}$ by the relation

$$
\pi(\mu)(g)=\mu(g \circ \varphi): g \in C(\mathbb{T})
$$

where $C(\mathbb{T})$ denotes the space of continuous real-valued functions defined on $\mathbb{T}$. Ditor and Eifler demonstrate that when $\varphi$ is continuous, onto and open, the map $\pi: P(\mathbb{S}) \longrightarrow P(\mathbb{T})$ is also a continuous, onto and open mapping with respect to the weak* topologies for both $P(\mathbb{S})$ and $P(\mathbb{T})$. Subsequently the open mapping theorem of Ditor and Eifler is generalized to Polish spaces by Eifler [5] and to Souslin spaces by Schief $[7$.

It is our object in the present paper to generalize the open mapping theorem on probability spaces derived by Ditor and Eifler to the spaces of Young measures on compact metric spaces. The notion of Young measures is introduced, first, by L. C. Young [9] to represent the "generalized solutions" in the relaxation problem of optimal control and the calculus of variation. It generalizes the notion of functions and describes the limit oscillation behavior of a sequence of functions. This notion is also known as a transition probability, a stochastic kernel, or a parametrized measure and is applied in a wide range of research. The theory of Young measures is also crucial to mathematical economics (see, e.g., Balder [2], Milgrom-Weber [6]).

Following the "Preliminaries" section, we demonstrate, in Section 3, that the open mapping theorem holds on the spaces of Young measures on compact metric spaces.

\section{Preliminaries}

Let $(\Omega, \mathcal{F}, \mu)$ be a complete finite positive measure space and let $\mathbb{S}$ be a Polish space with its Borel $\sigma$-algebra $\mathcal{B}(\mathbb{S})$. Denote by $P(\mathbb{S})$ the space of probability measures on $(\mathbb{S}, \mathcal{B}(\mathbb{S})) . \quad P(\mathbb{S})$ is endowed with the weak* topology, that is, the coarsest topology which makes the maps continuous:

$$
\nu \longmapsto \nu(\psi)=\int_{\mathbb{S}} \psi d \nu: \psi \in C^{b}(\mathbb{S}),
$$

where $C^{b}(\mathbb{S})$ denotes the set of continuous bounded real-valued functions defined on $\mathbb{S}$. A Young measure is a measure on $(\Omega \times \mathbb{S}, \mathcal{F} \otimes \mathcal{B}(\mathbb{S}))$ whose projection on $\Omega$ is equal to $\mu$. By the theorem of disintegration of measures, there corresponds, for each Young measure $\lambda$, a function $\nu: \Omega \times \mathcal{B}(\mathbb{S}) \longrightarrow[0,1]$, called a disintegration of measure $\lambda$, which satisfies the following conditions:

(i) $\nu(\omega, \cdot)$ is a probability measure for each $\omega \in \Omega$,

(ii) $\nu(\cdot, A)$ is measurable for each $A \in \mathcal{B}(\mathbb{S})$,

(iii) $\lambda(A)=\int_{\Omega}\left[\int_{\mathbb{S}} \chi_{A}(\omega, s) \nu_{\omega}(d s)\right] \mu(d \omega)$ for each $A \in \mathcal{F} \otimes \mathcal{B}(\mathbb{S})$,

where $\chi_{A}$ is a characteristic function of $A$. Note that condition (ii) is equivalent to the condition that the map $\omega \longmapsto \nu_{\omega}(\cdot): \Omega \longrightarrow P(\mathbb{S})$ is measurable with respect to the Borel $\sigma$-algebra generated by the weak* topology on $P(\mathbb{S})$. Note also that for each Young measure there correspond many disintegrations. However, there is exactly one one-to-one correspondence between the space of Young measures and the equivalence classes of $\mu$-a.e. equal disintegrations. We shall identify hereafter a 
Young measure with its disintegrations. We denote by $\mathcal{Y}(\Omega ; \mathbb{S})$ the set of all Young measures on $\Omega \times \mathbb{S}$.

A real-valued function $\psi$ defined on $\Omega \times \mathbb{S}$ is said to be a Carathéodory integrand if the following conditions hold:

(i) $\psi$ is $\mathcal{F} \otimes \mathcal{B}(\mathbb{S})$-measurable,

(ii) for each $\omega \in \Omega$, the map $s \longmapsto \psi(\omega, s)$ is continuous and bounded,

(iii) the map $\omega \longmapsto\|\psi(\omega, \cdot)\|_{\infty}$ is $\mu$-integrable,

where $\|\cdot\|_{\infty}$ stands for the sup-norm. The set of all Carathéodory integrands on $\Omega \times \mathbb{S}$ is denoted by $\mathcal{G}_{C}(\Omega ; \mathbb{S})$. The space $\mathcal{Y}(\Omega ; \mathbb{S})$ of Young measures is endowed with the coarsest topology which makes the maps continuous:

$$
\nu \longmapsto \nu(\psi)=\int_{\Omega \times \mathbb{S}} \psi d \nu=\int_{\Omega}\left[\int_{\mathbb{S}} \psi(\omega, s) \nu_{\omega}(d s)\right] \mu(d \omega): \psi \in \mathcal{G}_{C}(\Omega ; \mathbb{S}) .
$$

This topology on $\mathcal{Y}(\Omega ; \mathbb{S})$ is called the narrow topology.

If $\mathbb{S}$ is a compact metric space, the set $\mathcal{G}_{C}(\Omega ; \mathbb{S})$ is isometrically isomorphic to the space $L_{1}(\Omega, C(\mathbb{S}))$ of Bochner integrable functions defined on $\Omega$ into the space $C(\mathbb{S})$ of continuous real-valued functions defined on $\mathbb{S}$. The dual of $L_{1}(\Omega, C(\mathbb{S}))$ is the linear space $L_{\infty}\left(\Omega, M^{b}(\mathbb{S})\right)$ of equivalence classes of scalarly measurable functions defined on $\Omega$ into the space $M^{b}(\mathbb{S})$ of bounded measures. We can consider the space $\mathcal{Y}(\Omega ; \mathbb{S})$ of Young measures as a subset of $L_{\infty}\left(\Omega, M^{b}(\mathbb{S})\right)$. Then the narrow topology on $\mathcal{Y}(\Omega ; \mathbb{S})$ is equal to the relative topology of the topology on $L_{\infty}\left(\Omega, M^{b}(\mathbb{S})\right)$ defined by the duality $\sigma\left(L_{\infty}, L_{1}\right)$.

Let $L_{0}(\Omega, \mathbb{S})$ denote the set of measurable functions defined on $\Omega$ into $\mathbb{S}$. To each $f \in L_{0}(\Omega, \mathbb{S})$ we associate a degenerate Young measure $\delta^{f}$ defined by $\delta_{\omega}^{f}=\delta_{f(\omega)}$, where $\delta_{a}$ denotes a Dirac measure the probability of which is concentrated at $a$. Remark that when $\mathbb{S}$ is a compact metric space, the set $\mathcal{Y}(\Omega ; \mathbb{S})$ endowed with the narrow topology is compact and metrizable. Furthermore, if $(\Omega, \mathcal{F}, \mu)$ is nonatomic, the set of all degenerate Young measures is dense in the set of Young measures $\mathcal{Y}(\Omega ; \mathbb{S})$ (see, e.g., Warga [8], Theorem IV, 2.6).

Let $(X, d)$ be a compact metric space and let $2^{X}$ be the set of all closed subsets of $X$. We denote by $h(A, B)$ the Hausdorff metric on $2^{X}$ defined by

$$
h(A, B)=\max \left\{\sup _{x \in A} d(x, B), \sup _{y \in B} d(y, A)\right\} .
$$

Note that $2^{X}$ endowed with the Hausdorff metric is a compact metric space.

\section{AN OPEN MAPPING THEOREM FOR YOUNG MEASURES}

Let $(\Omega, \mathcal{F}, \mu)$ be a nonatomic complete finite positive measure space and let $\mathbb{S}, \mathbb{T}$ be compact metric spaces. Let $\varphi$ be a continuous map defined on $\mathbb{S}$ onto $\mathbb{T}$. We consider the map $\pi: P(\mathbb{S}) \longrightarrow P(\mathbb{T})$ defined by

$$
\pi(\mu)(g)=\mu(g \circ \varphi): g \in C(\mathbb{T})
$$

and the map $\Pi: \mathcal{Y}(\Omega ; \mathbb{S}) \longrightarrow \mathcal{Y}(\Omega ; \mathbb{T})$ defined by the relation: for all $g \in \mathcal{G}_{C}(\Omega ; \mathbb{T})$,

$$
\int_{\Omega}\left[\int_{\mathbb{T}} g(\omega, t) \Pi(\nu)_{\omega}(d t)\right] \mu(d \omega)=\int_{\Omega}\left[\int_{\mathbb{S}} g(\omega, \varphi(s)) \nu_{\omega}(d s)\right] \mu(d \omega) .
$$

First of all, let us make clear the meaning of equation (3.1) and confirm that the mapping $\Pi$ is well-defined. Recall that the space of all Carathéodory integrands $\mathcal{G}_{C}(\Omega ; \mathbb{T})$ is isometrically isomorphic to the space $L_{1}(\Omega, C(\mathbb{T}))$ of Bochner integrable 
functions defined on $\Omega$ into $C(\mathbb{T})$. We identify the two spaces and define, for each fixed $\nu \in \mathcal{Y}(\Omega, \mathbb{S})$, a bounded linear functional $\Lambda_{\nu}$ defined on $L_{1}(\Omega, C(\mathbb{T}))$ by

$$
\Lambda_{\nu}(g)=\int_{\Omega}\left[\int_{\mathbb{S}} g(\omega, \varphi(s)) \nu_{\omega}(d s)\right] \mu(d \omega) .
$$

Since the dual space of $L_{1}(\Omega, C(\mathbb{T}))$ is the space $L_{\infty}\left(\Omega, M^{b}(\mathbb{T})\right)$, we deduce the existence of $\Pi(\nu) \in L_{\infty}\left(\Omega, M^{b}(\mathbb{T})\right)$ satisfying

$$
\Lambda_{\nu}(g)=\int_{\Omega}\left[\int_{\mathbb{T}} g(\omega, t) \Pi(\nu)_{\omega}(d t)\right] \mu(d \omega) .
$$

Furthermore, the $\Pi(\nu)$ defined in this way constitutes a Young measure, as the following lemma indicates.

Lemma 1. $\Pi(\nu) \in \mathcal{Y}(\Omega, \mathbb{T})$ for $\nu \in \mathcal{Y}(\Omega, \mathbb{S})$.

Proof. Assume to the contrary that $\Pi(\nu) \notin \mathcal{Y}(\Omega, \mathbb{T})$. Then since $\mathcal{Y}(\Omega, \mathbb{T})$ is a compact convex set in $L_{\infty}\left(\Omega, M^{b}(\mathbb{T})\right)$ with respect to the topology $\sigma\left(L_{\infty}, L_{1}\right)$, there exists, by the separation theorem, $\psi \in \mathcal{G}_{C}(\Omega ; \mathbb{T})$ and $\varepsilon>0$ such that for all $\lambda \in \mathcal{Y}(\Omega, \mathbb{T})$,

$$
\int_{\Omega}\left[\int_{\mathbb{T}} \psi(\omega, t) \Pi(\nu)_{\omega}(d t)\right] \mu(d \omega) \geqq \varepsilon+\int_{\Omega}\left[\int_{\mathbb{T}} \psi(\omega, t) \lambda_{\omega}(d t)\right] \mu(d \omega) .
$$

On the other hand, let us set $\lambda_{\omega}^{*}=\pi\left(\nu_{\omega}\right)$ for all $\omega \in \Omega$. Then, since $\pi$ is easily seen to be continuous with respect to the weak* topologies for both $P(\mathbb{S})$ and $P(\mathbb{T})$, $\lambda^{*} \in \mathcal{Y}(\Omega, \mathbb{T})$, and so, by (3.1),

$$
\begin{aligned}
\int_{\Omega}\left[\int_{\mathbb{T}} \psi(\omega, t) \Pi(\nu)_{\omega}(d t)\right] \mu(d \omega) & =\int_{\Omega}\left[\int_{\mathbb{S}} \psi(\omega, \varphi(s)) \nu_{\omega}(d s)\right] \mu(d \omega) \\
& =\int_{\Omega}\left[\int_{\mathbb{T}} \psi(\omega, t) \lambda_{\omega}^{*}(d t)\right] \mu(d \omega) .
\end{aligned}
$$

This is a contradiction.

Let us now state the main theorem of the paper:

Theorem 1. Let $(\Omega, \mathcal{F}, \mu)$ be a nonatomic complete finite positive measure space, and let $\mathbb{S}, \mathbb{T}$ be compact metric spaces. Assume that $\varphi: \mathbb{S} \longrightarrow \mathbb{T}$ is a continuous, onto and open mapping. Then $\Pi: \mathcal{Y}(\Omega ; \mathbb{S}) \longrightarrow \mathcal{Y}(\Omega ; \mathbb{T})$ is also a continuous, onto and open mapping.

Proof. The continuity of $\Pi$ is immediate from the relation (3.1). So, we shall begin by demonstrating that $\Pi$ is onto.

We have to show that for each $\lambda \in \mathcal{Y}(\Omega ; \mathbb{T})$, there exists $\nu \in \mathcal{Y}(\Omega ; \mathbb{S})$ such that $\lambda=\Pi(\nu)$. First, remark that since the map $\pi: P(\mathbb{S}) \longrightarrow P(\mathbb{T})$ defined by the relation

$$
\pi(\mu)(g)=\mu(g \circ \varphi): g \in C(\mathbb{T})
$$

is easily seen to be continuous, it has a measurable graph. Further, the inverse (multi-valued) map $\pi^{-1}: P(\mathbb{T}) \longrightarrow P(\mathbb{S})$ is certainly closed-valued. It follows that $\pi^{-1}$ is a closed-valued measurable correspondence (see, e.g., Aubin-Frankowska [1, Theorem 8.14) and hence admits a measurable selection $\widehat{\pi}$ thanks to the measurable selection theorem (see, e.g., Aubin-Frankowska 11, Theorem 8.1.3). We define, for each $\omega \in \Omega, \nu_{\omega} \in P(\mathbb{S})$ by $\nu_{\omega}=\widehat{\pi}\left(\lambda_{\omega}\right)$. Then, since both the maps $\omega \longmapsto \lambda_{\omega}(\cdot)$ and $\widehat{\pi}$ are measurable, the map $\omega \longmapsto \nu_{\omega}(\cdot)$ is also measurable. Thus, we conclude 
that $\nu \in \mathcal{Y}(\Omega ; \mathbb{S})$. Also, for each $g \in \mathcal{G}_{C}(\Omega ; \mathbb{T})$ and $\omega \in \Omega$, we have, by the relation $\pi\left(\nu_{\omega}\right)=\lambda_{\omega}$, that

$$
\int_{\mathbb{T}} g(\omega, t) \lambda_{\omega}(d t)=\int_{\mathbb{S}} g(\omega, \varphi(s)) \nu_{\omega}(d s) .
$$

By integrating both sides of the above equality on $\Omega$ and taking into account relation (3.1), we obtain $\lambda=\Pi(\nu)$, and this completes the proof that $\Pi$ is onto.

Now we show the remaining property of $\Pi$; that is, $\Pi$ is open! Since $\mathcal{Y}(\Omega ; \mathbb{S})$ is compact and metrizable, we have only to show that for every $\nu \in \mathcal{Y}(\Omega ; \mathbb{S})$ and every compact neighborhood $U$ of $\nu$, the image $\Pi(U)$ is a neighborhood of $\lambda=\Pi(\nu)$ in $\mathcal{Y}(\Omega ; \mathbb{T})$. We assume contrarily that for some $\nu^{0} \in \mathcal{Y}(\Omega ; \mathbb{S})$ and some compact neighborhood $U$ of $\nu^{0}$, the set $\Pi(U)$ is not a neighborhood of $\lambda^{0}=\Pi\left(\nu^{0}\right)$. Remark that since $\Pi$ is continuous, $\Pi(U)$ is closed. Furthermore, by virtue of the nonatomicity of $(\Omega, \mathcal{F}, \mu)$, the space $L_{0}(\Omega, \mathbb{T})$ of measurable functions defined on $\Omega$ into $\mathbb{T}$ is dense in $\mathcal{Y}(\Omega ; \mathbb{T})$. Hence, there exists a sequence $\left\{f_{n}\right\} \subset L_{0}(\Omega, \mathbb{T})$ with $\delta^{f_{n}} \notin \Pi(U)$ such that $\delta^{f_{n}} \longrightarrow \lambda^{0}$ with respect to the narrow topology on $\mathcal{Y}(\Omega ; \mathbb{T})$. Set $A_{n}=\Pi^{-1}\left(\delta^{f_{n}}\right)$. Then, since $\mathcal{Y}(\Omega ; \mathbb{S})$ is compact and $A_{n}$ is compact and convex, there exists a subsequence $A_{n^{\prime}}$ of $A_{n}$ and a nonempty compact set $A \subset \mathcal{Y}(\Omega ; \mathbb{S})$ such that $h\left(A_{n^{\prime}}, A\right) \longrightarrow 0$ as $n^{\prime} \longrightarrow \infty$, where $h$ denotes the Hausdorff metric defined by any metric compatible with the narrow topology of $\mathcal{Y}(\Omega ; \mathbb{S})$.

We have now to show that the set $A$ is convex with $\nu^{0} \notin A$. As for the convexity of $A$, remark that $\mathcal{Y}(\Omega ; \mathbb{S})$ can be considered as a closed subset of a locally convex topological vector space $L_{\infty}\left(\Omega, M^{b}(\mathbb{S})\right)$, the topology of which is generated by a family of seminorms: $\nu \longmapsto|\nu(\psi)|$, where $\psi \in \mathcal{G}_{C}(\Omega ; \mathbb{S})$. Thus, the convexity of $A$ is a direct consequence of Theorem II.13 of Castaing-Valadier [3].

The relation $\nu^{0} \notin A$ can also be easily checked as follows. Assume to the contrary that $\nu^{0} \in A$. Then, since $\nu^{0}$ is an interior point of $U$ and $A_{n} \longrightarrow A$ with respect to the Hausdorff metric $h$, the set $A_{n}$ for all sufficiently large $n$ also contains an interior point of $U$. This is a contradiction to the definition of $A_{n}$.

Thus, by the separation theorem, we deduce the existence of $\psi \in \mathcal{G}_{C}(\Omega, \mathbb{S})$ and $\varepsilon>0$ such that

$$
\int_{\Omega \times \mathbb{S}} \psi d \nu^{0} \geqq \varepsilon+\int_{\Omega \times \mathbb{S}} \psi d \nu \quad \text { for all } \nu \in A .
$$

Let us now define a function $\psi^{*}: \Omega \times \mathbb{T} \longrightarrow \mathbb{R}$ by

$$
\psi^{*}(\omega, t)=\sup \left\{\psi(\omega, s): s \in \varphi^{-1}(t)\right\} .
$$

Then, since $\varphi$ is continuous and open by assumption, the correspondence $\varphi^{-1}(\cdot)$ is continuous. Further, since $\mathbb{S}$ is compact, $\varphi^{-1}(\cdot)$ is compact-valued. Hence, the continuity of $\psi(\omega, \cdot)$ implies, by Berge's maximum theorem, that $\psi^{*}$ is continuous in $t$. Also, $\psi^{*}$ is easily seen to be measurable in $\omega$ (see, e.g., Aubin-Frankowska [1], Theorem 8.2.11). Moreover, since $\left\|\psi^{*}(\omega, \cdot)\right\|_{\infty} \leqq\|\psi(\omega, \cdot)\|_{\infty}$ holds and $\psi$ is a Carathéodory integrand, the map $\omega \longmapsto\left\|\psi^{*}(\omega, \cdot)\right\|_{\infty}$ is $\mu$-integrable. Consequently, the map $\psi^{*}$ is a Carathéodory integrand.

The map $\Psi_{n}: \Omega \longrightarrow \mathbb{S}$ defined by

$$
\Psi_{n}(\omega)=\left\{s \in \varphi^{-1}\left(f_{n}(\omega)\right): \psi^{*}\left(\omega, f_{n}(\omega)\right)=\psi(\omega, s)\right\}
$$

is closed-valued and measurable. By having recourse to the measurable selection theorem again, we have a measurable map $g_{n}: \Omega \longrightarrow \mathbb{S}$ satisfying the following 
conditions:

$$
g_{n}(\omega) \in \varphi^{-1}\left(f_{n}(\omega)\right), \psi\left(\omega, g_{n}(\omega)\right)=\psi^{*}\left(\omega, f_{n}(\omega)\right) .
$$

By the first relation, we have $\Pi\left(\delta^{g_{n}}\right)=\delta^{f_{n}}$. Furthermore, by integrating the second, we have

$$
\int_{\Omega} \psi\left(\omega, g_{n}(\omega)\right) \mu(d \omega)=\int_{\Omega} \psi^{*}\left(\omega, f_{n}(\omega)\right) \mu(d \omega) .
$$

Now, since $\mathcal{Y}(\Omega ; \mathbb{S})$ is compact, we have a limit point $\nu^{*}$ of $\delta^{g_{n}}$. Further, since $\delta^{g_{n}} \in A_{n}$ for all $n$, we have $\nu^{*} \in A$. By taking limits in both sides of (3.3), we obtain the following estimates:

$$
\begin{aligned}
\int_{\Omega \times \mathbb{S}} \psi d \nu^{*} & =\int_{\Omega \times \mathbb{T}} \psi^{*} d \lambda^{0} \\
& =\int_{\Omega}\left[\int_{\mathbb{S}} \psi^{*}(\omega, \varphi(s)) \nu_{\omega}^{0}(d s)\right] \mu(d \omega) \\
& \geqq \int_{\Omega \times \mathbb{S}} \psi d \nu^{0},
\end{aligned}
$$

which contradicts (3.2) and completes the proof of the theorem.

\section{ACKNOWLEDGMENT}

The author expresses his gratitude to the referee for very helpful comments and suggestions.

\section{REFERENCES}

[1] J.-P. Aubin and H. Frankowska, Set-Valued Analysis, Birkhäuser, Boston, 1990. MR 1048347 (91d:49001)

[2] E. Balder, Generalized equilibrium results for games with incomplete information, Math. Oper. Res., 13(1988), 265-276. MR942618 (90d:90104)

[3] C. Castaing and M. Valadier, Convex Analysis and Measurable Multifunctions, Lect. Notes in Math., 580, Springer-Verlag, Berlin, 1977. MR0467310 (57:7169)

[4] S.Z. Ditor and L.Q. Eifler, Some open mapping theorems for measures, Trans. Amer. Math. Soc., 164(1972), 287-293. MR0477729 (57:17242)

[5] L.Q. Eifler, Open mapping theorems for probability measures on metric spaces, Pacific J. Math. 66(1976), 89-97. MR0453960 (56:12212b)

[6] P.R. Milgrom and R.J. Weber, Distributional strategies for games with incomplete information, Math. Oper. Res., 10(1985), 619-632. MR812820 (86m:90186)

[7] A. Schief, An open mapping theorem for measures, Monatsh. Math., 108(1989), 59-70. MR.1018825 (91c:46040)

[8] J. Warga, Optimal Control of Differential and Functional Equations, Academic Press, New York, 1972. MR0372708(51:8915)

[9] L.C. Young, Generalized curves and the existence of an attained absolute minimum, Calculus of Variations, C.R. Soc. Sc. Varsovie, 30(1937), 212-234.

School of Economics, Nagoya University, Furo-cho, Chikusa-ku, Nagoya, 464-8601, JAPAN

E-mail address: tateishi@soec.nagoya-u.ac.jp 